Bulletin of the Seismological Society of America. Vol. 57, No. 3, pp. 477-499. June, 1967

\title{
REFLECTION AND TRANSMISSION FROM A PLANE LAYERED CORE-MANTLE BOUNDARY
}

\author{
By Ta-Liang Teng
}

ABSTRACT

\begin{abstract}
A class of transfer functions in terms of layer matrices is derived, giving the transmission and reflection of plane harmonic $P$ or $S$ waves incident from either side of a plane layered core-mantle boundary. A computer program coded in complex arithmetic for the IBM 7094 is used to evaluate these functions. Numerical values obtained from five suggested models of the core-mantle boundary are compared and discussed. The aim is to formulate the method, and to establish some general guide, for the studies of the structure of the core-mantle boundary and the attenuation of seismic waves inside the core.
\end{abstract}

\section{INTRODUCTION}

Previously, Gutenberg (1959) had proposed that there is a slight decrease in velocity at the base of the mantle (Region $D^{\prime \prime}$ ). The standard solutions for the lower few hundred $\mathrm{km}$ of the mantle also suggest that it is at least "mildly inhomogeneous" (Bullen, 1963). Two recent studies, with different approaches, have lent further support to the earlier speculations. On the one hand, from the disagreement between a number of theoretical and observed free periods of the earth's spheroidal oscillation, Dorman et al (1966) have raised some doubt about the sharpness of the core-mantle boundary. They have noted that this disagreement can be reconciled by postulating a slightly larger core surrounded by a thin soft layer at the base of the mantle. On the other hand, Phinney and Alexander, 1966; also Alexander and Phinney, 1966 using compressional waves from large earthquakes observed deep inside the shadow zone, calculated spectral ratios and interpreted them in terms of a diffraction theory which they advanced to include a multilayered core-mantle geometry. Their analysis suggested that a layer at the base of the mantle with thickness in the approximate range $30-160 \mathrm{~km}$ would fit their data.

The present study makes use of the core phases and interprets them in terms of reflection and transmission at a plane layered core-mantle boundary. This approach is encouraged by the usefulness of the crustal transfer functions in the studies of layered crustal structure (Haskell, 1960, 1962; Phinney, 1964; Fernandez, 1965; among others), and this has recently been attempted by Kanamori (1966). Using spectrums of short-period $P$ and $P c P$ waves, he designed an experiment to measure the $Q$ of the mantle, and at the same time to investigate a possible transitional layer at the core-mantle boundary. Since a short time window $(\sim 5 \mathrm{sec})$ was used, he was able to measure $Q$ and avoid the complexity due to reflections from a possible layered core-mantle boundary. On the other hand, just because a narrow time window can not adequately include the later arrivals from reverberations caused by the layered structure, his data is not sensitive for the detection of a transitional layer. This insensitivity can also be demonstrated in the frequency domain. If one convolves the core-mantle transfer function with a function corresponding to the 
narrow time window, the resulting slowly varying function would give no clear characteristics by which a transitional core-mantle structure can be distinguished from a sharp one.

In order to broaden the application of the transfer function technique to various core phases, the present study first derives a complete class of the complex transfer functions. Both transmission and reflection from $P_{-}, S V$-, and $S H$-type incident plane waves at the plane layered core-mantle boundary are considered. A computer program is prepared to evaluate this class of complex transfer functions. Numerical. values of these functions for five suggested models of the core-mantle boundary are obtained and they are discussed within the framework of the following questions:

(1) for an assumed structure of the core-mantle boundary, what effects on core phases can be expected and which core phases are more sensitive to the layered structure?

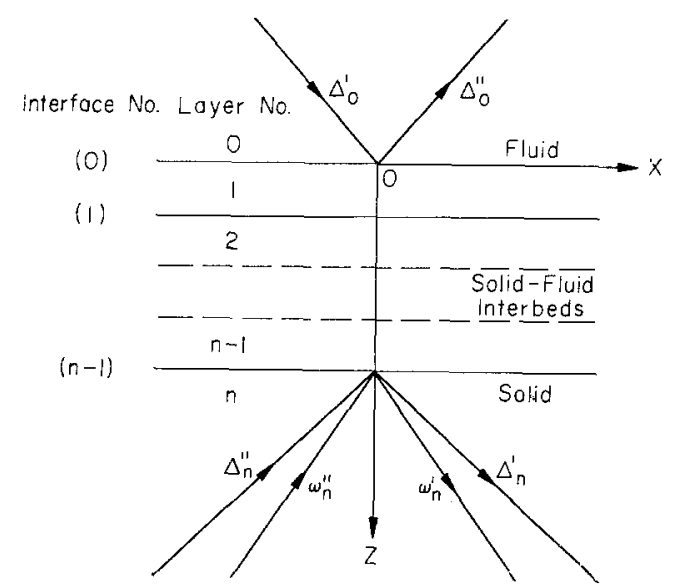

Fig. 1. Geometry of the problem of plane-wave transmission and reflection at a layered boundary.

(2) at what epicentral distances do these effects become more pronounced?

(3) what frequency bands (or records from what instruments) are most suitable to detect these effects?

(4) what window length is best suitable for a study of the core-mantle boundary? In addition, the above numerical results have also defined the regions (in terms of epicentral distances, frequency bands, and types of core phases) in which the aforementioned effects become small. Consequently, even without detailed knowledge about the core-mantle boundary, one can still make good use of certain core phases to study the attenuation of the earth, particularly the portion inside the core.

\section{THEORY}

Consider an infinite space (Figure 1) composed of an upper fluid half-space, a lower solid half-space, and, in between, a number of arbitrarily interbedded solid and fluid layers. Number the fluid half-space the zeroth layer which rests on the zeroth interface, and so on down with the $(n-1)^{\text {th }}$ interface overlying the $n^{\text {th }}$ layer, or the solid half-space. All interfaces are parallel. 
Continuity of two displacement components and two stress components at each solid-solid interface, and of vertical displacement and normal stress at either solidfluid or fluid-fluid interface give rise to the following transformations:

$$
\left[\begin{array}{c}
\Delta_{n}^{\prime}+\Delta_{n}^{\prime \prime} \\
\Delta_{n}^{\prime}-\Delta_{n}^{\prime \prime} \\
\omega_{n}^{\prime}-\omega_{n}^{\prime \prime} \\
{\omega_{n}}^{\prime}+\omega_{n}^{\prime \prime}
\end{array}\right]=E_{n}{ }^{-1} A\left[\begin{array}{c}
\dot{u}_{r} / c \\
\dot{w}_{0} / c \\
\sigma_{0} \\
0
\end{array}\right]
$$

where

$\Delta_{n}, \omega_{n}=$ the dilatational and rotational amplitudes of $P$ and $S V$ waves in the solid half-space. The single primes represent down-going waves and the double primes represent up-going waves.

$E_{n}^{-1}=$ the matrix defined by Haskell (1953, equations 2-16).

$\dot{u}_{r}=$ the horizontal particle velocity at the first solid-fluid interface above the solid half-space.

$\dot{w}_{0}=$ the vertical particle velocity at the zeroth interface.

$\sigma_{0}=$ the normal stress at the zeroth interface.

$c=$ apparent horizontal velocity.

$A$ is a product of $n-1$ matrices of the form $a_{m}$ for a solid layer and $a_{m}{ }^{\prime}$ for a fluid layer. The elements of $a_{m}$ are given in Haskell (1953, p. 21), and $a_{m}{ }^{\prime}$ has the form (see Appendix A)

$$
a_{m}^{\prime}=\left[\begin{array}{cccc}
1 & 0 & 0 & 0 \\
0 & \cos P_{m} & i r_{\alpha_{m}}\left(\rho_{m} c^{2}\right)^{-1} \sin P_{m} & 0 \\
0 & i \rho_{m} c^{2} r_{\alpha_{m}}^{-1} \sin P_{m} & \cos P_{m} & 0 \\
0 & 0 & 0 & 1
\end{array}\right]
$$

where

$$
\begin{aligned}
\rho_{m} & =\text { density } \\
r_{\alpha_{m}} & = \begin{cases}{\left[\left(c / \alpha_{m}\right)^{2}-1\right]^{1 / 2}} & c>\alpha_{m} \\
-i\left[1-\left(c / \alpha_{m}\right)^{2}\right]^{1 / 2} & c<\alpha_{m}\end{cases} \\
P_{m} & =k r_{\boldsymbol{\alpha}_{m}} d_{m} \\
k & =\omega / c \\
d_{m} & =\text { thickness. }
\end{aligned}
$$

As an example, if $n=5$, there are four layers between the two half-spaces. Let layer $1,2,3$, and 4 be fluid, solid, fluid and solid respectively. Then $A$ takes the following form

$$
A=a_{4} a_{3}{ }^{\prime} a_{2} a_{1}{ }^{\prime}
$$

For the fluid half space, the vector $\left(0, \dot{w}_{0} / c, \sigma_{0}, 0\right)$ and the dilatations $\Delta_{0}^{\prime}$ and $\Delta_{0}^{\prime \prime}$ are related by (see Appendix $A$ ) 


$$
\left[\begin{array}{c}
0 \\
\frac{\dot{w}_{-1}}{c} \\
\sigma_{-1} \\
0
\end{array}\right]=E_{0}{ }^{\prime}\left[\begin{array}{c}
\Delta_{0}^{\prime}+\Delta_{0}^{\prime \prime} \\
\Delta_{0}^{\prime}-\Delta_{0}^{\prime \prime} \\
0 \\
0
\end{array}\right]
$$

where

$\dot{w}_{-1}=$ the vertical particle velocity in the fluid half-space

$\sigma_{-1}=$ the normal stress in the fluid half-space

$$
E_{0}^{\prime}=\left[\begin{array}{cccc}
0 & 0 & 0 & 1 \\
0 & -\left(\alpha_{0} / c\right)^{2} r_{\alpha_{0}} & 0 & 0 \\
\rho_{0} \alpha_{0}^{2} & 0 & 0 & 0 \\
0 & 0 & 1 & 0
\end{array}\right]
$$

Continuity of vertical particle velocity and normal stress at the zeroth interface results from (1) and (3) in the following relations

$$
\begin{aligned}
& \Delta_{n}{ }^{\prime}+\Delta_{n}{ }^{\prime \prime}=J_{11} \frac{\dot{u}_{r}}{c}+J_{12} \frac{\dot{w}_{0}}{c}+J_{13} \sigma_{0} \\
& \Delta_{n}{ }^{\prime}-\Delta_{n}{ }^{\prime \prime}=J_{21} \frac{\dot{u}_{r}}{c}+J_{22} \frac{\dot{w}_{0}}{c}+J_{23} \sigma_{0} \\
& \omega_{n}^{\prime}-\omega_{n}^{\prime \prime}=J_{31} \frac{\dot{u}_{r}}{c}+J_{32} \frac{\dot{w}_{0}}{c}+J_{33} \sigma_{0} \\
& \omega_{n}^{\prime}+\omega_{n}^{\prime \prime}=J_{4 \mathrm{1}} \frac{\dot{u}_{r}}{c}+J_{42} \frac{\dot{w}_{0}}{c}+J_{43} \sigma_{0} \\
& \Delta_{0}^{\prime}+\Delta_{0}^{\prime \prime}=\sigma_{0} /\left(\rho_{0} \alpha_{0}{ }^{2}\right) \\
& \Delta_{0}^{\prime}-\Delta_{0}^{\prime \prime}=-\dot{w}_{0} c /\left(\alpha_{0}{ }^{2} r_{\alpha_{0}}\right)
\end{aligned}
$$

where

$$
\left(J_{i j}\right)=J=E_{n}^{-1} A \text {. }
$$

This system of linear equations is used to solve for the reflection and transmission coefficients for different incident wave types from either half-space.

Incident $P$ waves from solid half space. Setting $\Delta_{0}^{\prime \prime}=\omega_{n}^{\prime \prime}=0$ and eliminating $\dot{u}_{r}, \dot{w}_{0}, \sigma_{0}$ from (5), we find $[1] P-P$ reflection coefficient, $S_{r}{ }^{P P}$, due to a $P$-wave 
incident from the solid half-space. (The same expression has been given by Kanamori, 1966).

$$
\begin{aligned}
S_{r}{ }^{P P}=\frac{\Delta_{n}{ }^{\prime}}{\Delta_{n}^{\prime \prime}}=D^{-1}\{ & {\left[\left(J_{12}+J_{22}\right)\left(J_{31}-J_{41}\right)-\left(J_{11}+J_{21}\right)\left(J_{32}-J_{42}\right)\right] \cdot p } \\
+ & {\left.\left[\left(J_{13}+J_{23}\right)\left(J_{31}-J_{41}\right)-\left(J_{11}+J_{21}\right)\left(J_{33}-J_{43}\right)\right] q\right\} . }
\end{aligned}
$$

[2] P-P transmission coefficient, $S_{t}{ }^{P}$, due to a $P$-wave incident from the solid halfspace

$$
S_{l}^{P P}=\frac{\Delta_{0}^{\prime}}{\Delta_{n}^{\prime \prime}}=2 D^{-1}\left(J_{31}-J_{41}\right)
$$

[3] $P$-SV reflection coefficient, $S_{r}^{P S V}$, due to a $P$-wave incident from the solid halfspace

$$
S_{r}^{P S V}=\frac{\omega_{n}^{\prime}}{\Delta_{n}^{\prime \prime}}=2 D^{-1}\left[\left(J_{31} J_{42}-J_{32} J_{41}\right) p+\left(J_{31} J_{43}-J_{33} J_{41}\right) q\right]
$$

where

$$
\begin{aligned}
& D=\left[-\left(J_{11}-J_{21}\right)\left(J_{32}-J_{42}\right)+\left(J_{12}-J_{22}\right)\left(J_{31}-J_{41}\right)\right] p \\
& +\left[-\left(J_{11}-J_{21}\right)\left(J_{33}-J_{43}\right)+\left(J_{13}-J_{23}\right)\left(J_{31}-J_{41}\right)\right] q \\
& p=\left(\alpha_{0} / c\right)^{2} r_{\alpha_{0}} \\
& q=\rho_{0} \alpha_{0}^{2} .
\end{aligned}
$$

Incident $S V$ waves from solid half-space. Setting ${\Delta_{n}}^{\prime \prime}={\Delta_{0}}^{\prime}=0$ and eliminating $\dot{u}_{r}, \dot{w}_{0}, \sigma_{0}$ from (5), we find [4] $S V-S V$ reflection coefficient, $S_{r}^{S V S V}$, due to an $S V$-wave incident from the solid half-space

$$
\begin{aligned}
S_{r}{ }^{S V S V}=\frac{\omega_{n}^{\prime}}{\omega_{n}{ }^{\prime \prime}}=D^{-1} & \left\{\left(J_{21}-J_{11}\right)\left(J_{32}+J_{42}\right)+\left(J_{12}-J_{22}\right)\left(J_{31}+J_{41}\right)\right] p \\
+ & {\left.\left[\left(J_{21}-J_{11}\right)\left(J_{33}+J_{43}\right)+\left(J_{13}-J_{23}\right)\left(J_{31}+J_{41}\right)\right] q\right\} }
\end{aligned}
$$

[5] $S V-P$ transmission coefficient, $S_{t}{ }^{S V P}$, due to an $S V$-wave incident from the solid half-space

$$
S_{t}^{S V P}=\frac{\Delta_{0}^{\prime \prime}}{\omega_{n}^{\prime \prime}}=2 D^{-1}\left(J_{21}-J_{11}\right)
$$

[6] $S V-P$ reflection coefficient, $S_{\mathrm{r}}{ }^{S V P}$, due to an $S V$-wave incident from the solid half-space

$$
S_{r}^{S V P}=\frac{\Delta_{n}^{\prime}}{\omega_{n}{ }^{\prime \prime}}=2 D^{-1}\left[\left(J_{12} J_{21}-J_{11} J_{22}\right) p+\left(J_{13} J_{21}-J_{11} J_{23}\right) q\right] .
$$


Incident $S H$ waves from the solid half-space. [7] $S H$-SH reflection coefficient, $S_{r}{ }^{S H S H}$

$$
S_{r}^{S H S H}=\frac{\mu_{n} r_{\beta_{n}} A_{11}-A_{21}}{\mu_{n} r_{\beta_{n}} A_{11}+A_{21}}
$$

where

$$
\begin{aligned}
r_{\beta_{n}} & =\left\{\begin{array}{l}
{\left[\left(c / \beta_{n}\right)^{2}-1\right]^{1 / 2} \quad c>\beta_{n}} \\
-i\left[1-\left(c / \beta_{n}\right)^{2}\right]^{1 / 2} \quad c<\beta_{n}
\end{array}\right. \\
\mu_{n} & =\text { rigidity of the solid half-space } \\
A_{i j} & =\prod_{n=1}^{n-1} a_{m}, \text { and } a_{m} \text { is given by equation } 3 \text { of Haskell }(1960) .
\end{aligned}
$$

The amplitude part of (13) is trivial: $\left|S_{r}{ }^{S H}{ }^{S H}\right| \equiv 1$; as required by energy conservation, total reflection must occur at the first solid-fluid interface.

Incident $P$ waves from the fluid half-space. Similarly, setting $\Delta_{n}^{\prime \prime}=\omega_{n}^{\prime \prime}=0$ and eliminating $\dot{u}_{r}, \dot{u}_{0}, \sigma_{0}$ from (5), we find $[8] P-P$ reflection coefficient, $F_{r}{ }^{P}$, due to a $P$-wave incident from the fluid half-space

$$
\begin{aligned}
F_{r}{ }^{P}=\frac{\Delta_{0}^{\prime \prime}}{\Delta_{0}^{\prime}}=D^{-1} & \left\{\left[-\left(J_{11}-J_{21}\right)\left(J_{32}-J_{42}\right)+\left(J_{12}-J_{22}\right)\left(J_{31}-J_{41}\right)\right] p\right. \\
- & {\left.\left[-\left(J_{11}-J_{21}\right)\left(J_{33}-J_{43}\right)+\left(J_{13}-J_{23}\right)\left(J_{31}-J_{41}\right)\right] q\right\} }
\end{aligned}
$$

[9] P-P transmission coefficient, $F_{t}{ }^{P}$, due to a $P$-wave incident from the fluid halfspace

$$
\begin{aligned}
F_{t}{ }^{P P}=\frac{\Delta_{n}{ }^{\prime}}{\Delta_{0}{ }^{\prime}} & =2 p q D^{-1}\left[\left(J_{31}-J_{41}\right)\left(J_{12} J_{23}-J_{13} J_{22}\right)\right. \\
& \left.+\left(J_{32}-J_{42}\right)\left(J_{13} J_{21}-J_{11} J_{23}\right)+\left(J_{33}-J_{43}\right)\left(J_{11} J_{22}-J_{12} J_{21}\right)\right]
\end{aligned}
$$

[10] $P$-SV transmission coefficient, $F_{t}{ }^{P S V}$, due to a $P$-wave incident from the fluid half-space

$$
\begin{aligned}
F_{t}{ }^{P S V}= & \frac{\omega_{5}{ }^{\prime}}{\Delta_{0}{ }^{\prime}}=2 p q D^{-1}\left[\left(J_{11}-J_{21}\right)\left(J_{33} J_{42}-J_{32} J_{43}\right)\right. \\
& \left.+\left(J_{12}-J_{22}\right)\left(J_{31} J_{43}-J_{33} J_{41}\right)+\left(J_{13}-J_{23}\right)\left(J_{32} J_{41}-J_{31} J_{42}\right)\right] .
\end{aligned}
$$

\section{Energy Relationships}

For an arbitrary number of plane parallel layers, the energy flow rate $\epsilon_{n-1}$, across the $(n-1)^{\text {th }}$ interface (Figure 1) can be calculated by

$$
\epsilon_{n-1}=R\left(\dot{u}_{n-1} \tau_{n-1}^{*}+\dot{w}_{n-1} \sigma_{n-1}^{*}\right)
$$

where $R$ indicates the real part of the complex quantity that follows. This can be shown to equal (Haskell, 1962).

$$
\begin{aligned}
\epsilon_{n-1}=\left(\rho_{n} \alpha_{n}{ }^{4} / c\right)\left(\left|\Delta_{n}{ }^{\prime \prime}\right|^{2}-\left|\Delta_{n}{ }^{\prime}\right|^{2}\right) R\left(r_{\alpha_{n}}\right) & \\
& +4\left(\rho_{n} \beta_{n}{ }^{4} / c\right)\left(\left|\omega_{n}{ }^{\prime \prime}\right|^{2}-\left|\omega_{n}{ }^{\prime}\right|^{2}\right) r_{\beta_{n}} .
\end{aligned}
$$


Likewise, the energy flow rate, $\epsilon_{-1}$, across the zeroth interface can be calculated by

$$
\epsilon_{-1}=R\left(\dot{u}_{-1} \tau_{-1}^{*}+\dot{w}_{-1} \sigma_{-1}^{*}\right) .
$$

If the zeroth layer is fluid

$$
\epsilon_{-1}=R\left(\dot{w}_{-1} \sigma_{-1}^{*}\right)
$$

From (3) and (4) we have

$$
\begin{aligned}
\dot{w}_{-1} & =-\left(\alpha_{0}^{2} r_{\alpha_{0}} / c\right)\left(\Delta_{0}^{\prime}-\Delta_{0}^{\prime \prime}\right) \\
\sigma_{-1}^{*} & =\rho_{0} \alpha_{0}^{2}\left(\Delta_{0}^{\prime *}+\Delta_{0}{ }^{\prime \prime *}\right) .
\end{aligned}
$$

Putting (20) into (19), we find

$$
\begin{aligned}
\epsilon_{-1}=-\left(\rho_{0} \alpha_{0}^{4} / c\right)\left\{R\left[r_{\alpha_{0}}\left(\left|\Delta_{0}^{\prime}\right|^{2}-\left|\Delta_{0}^{\prime \prime}\right|^{2}\right)\right]\right. & \\
& \left.+R\left[r_{\alpha_{0}}\left(\Delta_{0}^{\prime} \Delta_{0}^{\prime \prime *}-\Delta_{0}^{\prime *} \Delta_{0}^{\prime \prime}\right)\right]\right\} .
\end{aligned}
$$

Here, $\left(\left|\Delta_{0}^{\prime}\right|^{2}-\left|\Delta_{0}^{\prime \prime}\right|^{2}\right)$ is always real, and $\left(\Delta_{0}{ }^{\prime} \Delta_{0}^{\prime \prime *}-\Delta_{0}{ }^{\prime *} \Delta_{0}^{\prime \prime}\right)$ is always pure imaginary. For $c>\alpha_{0}, r_{\alpha_{0}}$ is real and the second term in the square brackets vanishes. But for $c<\alpha_{0}, r_{\alpha_{0}}$ is pure imaginary, the finiteness of the displacements at infinity requires that $\Delta_{0}^{\prime \prime}=0$, therefore, both terms in the square brackets vanish. This latter case corresponds to a total reflection at the zeroth interface across which no energy transmits, or $\epsilon_{-1}=0$. The former case is more interesting, corresponding to wave reflection and transmission. The partition of energy among these reflected and transmitted waves is to be discussed in the following.

If the medium is non-dissipative, by the law of energy conservation we have

$$
\begin{aligned}
\left(\rho_{0} \alpha_{0}{ }^{4} / c\right)\left(\left|\Delta_{0}{ }^{\prime}\right|^{2}-\left|\Delta_{0}{ }^{\prime \prime}\right|^{2}\right) r_{\alpha_{0}}{ }^{\prime}= & \left(\rho_{n} \alpha_{n}{ }^{4} / c\right)\left(\left|\Delta_{n}{ }^{\prime \prime}\right|^{2}-\left|\Delta_{n}{ }^{\prime}\right|^{2}\right) R\left(r_{\alpha_{n}}\right) \\
& +4\left(\rho_{n} \beta_{n}{ }^{4} / c\right)\left(\left|\omega_{n}{ }^{\prime \prime}\right|^{2}-\left|\omega_{n}{ }^{\prime}\right|^{2}\right) r_{\beta_{n}} .
\end{aligned}
$$

For the $P$-type incident waves from the solid half-space, $\left(\Delta_{0}^{\prime}=\omega_{n}^{\prime \prime}=0\right)$ the partition of energy among the reflected and transmitted $P$ and $S$ waves is given from (7), (8) and (9) by

$$
\begin{aligned}
E_{S_{r}}^{P P}=\left|\Delta_{n}{ }^{\prime} / \Delta_{n}{ }^{\prime \prime}\right|^{2}=\mid D^{-1}\left\{\left[\left(J_{12}+J_{22}\right)\left(J_{31}-J_{41}\right)\right.\right. & \left.\left.-\left(J_{11}+J_{21}\right)\left(J_{33}-J_{43}\right)\right] q\right\}\left.\right|^{2} \\
\left.-\left(J_{11}+J_{21}\right)\left(J_{32}-J_{42}\right)\right] p+ & {\left[\left(J_{13}+J_{23}\right)\left(J_{31}-J_{41}\right)\right.} \\
E_{S_{t}}^{P P}= & {\left[\left(\rho_{0} \alpha_{0}^{4} r_{\alpha_{0}}\right) /\left(\rho_{n} \alpha_{n}{ }^{4} r_{\alpha_{n}}\right)\right]\left|\Delta_{0}^{\prime \prime} / \Delta_{n}^{\prime \prime}\right|^{2} } \\
= & 4\left[\left(\rho_{0} \alpha_{0}^{4} r_{\alpha_{0}}\right) /\left(\rho_{n} \alpha_{n}{ }^{4} r_{\alpha_{n}}\right)\right]\left|D^{-1}\left(J_{31}-J_{41}\right)\right|^{2}
\end{aligned}
$$




$$
\begin{aligned}
E_{\delta_{r}}^{P S V} & =4\left[\left(\beta_{n}{ }^{4} r_{\beta_{n}}\right) /\left(\alpha_{n}{ }^{4} r_{\alpha_{n}}\right)\right]\left|\omega_{n}{ }^{\prime} / \Delta_{n}{ }^{\prime \prime}\right|^{2} \\
& =16\left[\left(\beta_{n}^{4} r_{\beta_{n}}\right) /\left(\alpha_{n}{ }^{4} r_{\alpha_{n}}\right)\right] \mid D^{-1}\left[\left(J_{31} J_{42}-J_{32} J_{41}\right) p\right. \\
& \left.+\left(J_{31} J_{43}-J_{33} J_{41}\right) q\right]\left.\right|^{2} .
\end{aligned}
$$

If the incident waves are of $S V$ type from the solid half-space, $\left(\Delta_{0}^{\prime}=\Delta_{n}{ }^{\prime \prime}=0\right)$, the partition of energy among the reflected and transmitted $P$ and $S$ waves is given from (10), (11), and (12) by

$$
\begin{aligned}
E_{S_{r}}^{S V S V}= & \left|\omega_{n}{ }^{\prime} / \omega_{n}{ }^{\prime \prime}\right|^{2}=\mid D^{-1}\left\{\left[\left(J_{21}-J_{11}\right)\left(J_{32}+J_{42}\right)\right.\right. \\
& \left.+\left(J_{12}-J_{2 \varepsilon}\right)\left(J_{31}+J_{41}\right)\right] p+\left[\left(J_{21}-J_{11}\right)\left(J_{23}+J_{43}\right)\right. \\
+ & \left.\left.+\left(J_{13}-J_{23}\right)\left(J_{31}+J_{41}\right)\right] q\right\}\left.\right|^{2} \\
E_{S_{i}}^{S V P}= & {\left[\left(\rho_{0} \alpha_{0}{ }^{4} r_{\alpha_{0}}\right) /\left(4 \rho_{n} \beta_{n}{ }^{4} r_{\alpha_{n}}\right)\right]\left|\Delta_{0}^{\prime \prime} / \omega_{n}^{\prime \prime}\right|^{2} } \\
= & {\left[\left(\rho_{0} \alpha_{0}{ }^{4} r_{\alpha_{0}}\right) /\left(\rho_{n} \beta_{n}{ }^{4} r_{\beta_{n}}\right)\right]\left|D^{-1}\left(J_{21}-J_{11}\right)\right|^{2} } \\
E_{S_{r}}^{S V P P}= & {\left[\left(\alpha_{n}{ }^{4} r_{\alpha_{n}}\right) /\left(4 \beta_{n}{ }^{4} r_{\beta_{n}}\right)\right]\left|\Delta_{n}{ }^{\prime} / \omega_{n}{ }^{\prime \prime}\right|^{2} } \\
= & {\left[\left(\alpha_{n}{ }^{4} r_{\alpha_{n}}\right) /\left(\beta_{n}{ }^{4} r_{\beta_{n}}\right)\right] \mid D^{-1}\left[\left(J_{12} J_{21}-J_{11} J_{22}\right) p\right.}
\end{aligned}
$$

If the incident waves are of $P$ type from the fluid half-space, $\omega_{n}{ }^{\prime}=\Delta_{n}{ }^{\prime \prime}=0$, the partition of energy among the reflected $P$ and the transmitted $P$ and $S$ waves is given from (14), (15), and (16) by

$$
\begin{gathered}
E_{F_{r}}^{P P}=\left|\Delta_{0}^{\prime \prime} / \Delta_{0}^{\prime}\right|^{2}=\mid D^{-1}\left\{\left[-\left(J_{11}-J_{21}\right)\left(J_{32}-J_{42}\right)+\left(J_{11}-J_{2 \varepsilon}\right)\right.\right. \\
\left.\cdot\left(J_{31}-J_{41}\right)\right] p-\left[-\left(J_{11}-J_{21}\right)\left(J_{33}-J_{43}\right)\right. \\
\begin{aligned}
E_{F^{\prime}}^{P P}=\left[\left(\rho_{n} \alpha_{n}{ }^{4} r_{\alpha_{n}}\right) /\left(\rho_{0} \alpha_{0}{ }^{4} r_{\alpha_{0}}\right)\right]\left|\Delta_{n}{ }^{\prime} / \Delta_{0}{ }^{\prime}\right|^{2} \\
=4\left[\left(\rho_{n} \alpha_{n}{ }^{4} r_{\alpha_{n}}\right) /\left(\rho_{0} \alpha_{0}{ }^{4} r_{\alpha_{0}}\right)\right] \mid p q D^{-1} \\
\left.\cdot\left[\left(J_{31}-J_{23}\right)\left(J_{31}-J_{41}\right)\right] q\right\}\left.\right|^{2}\left(J_{12} J_{23}-J_{13} J_{22}\right) \\
+\left(J_{32}-J_{42}\right)\left(J_{13} J_{21}-J_{11} J_{23}\right) \\
\left.+\left(J_{33}-J_{43}\right)\left(J_{11} J_{22}-J_{12} J_{21}\right)\right]\left.\right|^{2}
\end{aligned}
\end{gathered}
$$




$$
\begin{aligned}
& E_{F_{t}}^{P S}=4\left[\left(\rho_{n} \beta_{n}{ }^{4} r_{\beta_{n}}\right) /\left(\rho_{0} \alpha_{0}{ }^{4} r_{\alpha_{0}}\right)\right]\left|\omega_{n}{ }^{\prime} / \Delta_{0}{ }^{\prime}\right|^{2} \\
&=16\left[\left(\rho_{n} \beta_{n}{ }^{4} r_{\beta_{n}}\right) /\left(\rho_{0} \alpha_{0}{ }^{4} r_{\alpha_{0}}\right)\right] \mid p q D^{-1}\left[\left(J_{11}-J_{21}\right)\left(J_{33} J_{42}-J_{3 今} J_{43}\right)\right. \\
& \quad+\left(J_{12}-J_{22}\right)\left(J_{31} J_{43}-J_{33} J_{41}\right)+\left(J_{13}-J_{23}\right) \\
&\left.\cdot\left(J_{32} J_{41}-J_{31} J_{42}\right)\right]\left.\right|^{2} .
\end{aligned}
$$

The law of energy conservation (22) can be, therefore, written in more explicit form:

$$
\begin{aligned}
& E_{S_{r}}^{P P}+E_{S_{r}}^{P S V}+E_{S_{t}}^{P P}=1 \\
& E_{S_{r}}^{S Y S V}+E_{S_{r}}^{S Y P}+E_{S_{t}}^{S V P}=1 \\
& E_{F_{r}}^{P P}+E_{F^{r} t}^{P P}+E_{F_{t}}^{P S V}=1 .
\end{aligned}
$$

It can also be shown (Appendix B) that for equal values of $c$ the following relations exist:

$$
\begin{aligned}
& E_{S_{t}}^{P P}(c)=E_{F_{t}}^{P P}(c) \\
& E_{S_{r}}^{P S V}(c)=E_{S_{r}}^{S V P}(c)
\end{aligned}
$$

and

$$
E_{S_{t}}^{S V P}(c)=E_{F_{t}}^{P S V}(c) .
$$

By (32) and (33) it is therefore only necessary to calculate three distinct $E$ functions and the other six can be obtained through elementary operations. (33) remains true if both half spaces are solid.

\section{Numerical Results}

A Fortran program has been written in complex arithmetic for an IBM 7094 to evaluate the functions in equations (7) to (16). Numerical results have been obtained for a number of models, whose layer parameters are given in Table 1. These results provide a useful guidance in designing seismological experiments in the study of the earth's deep interior. From these numerical results based on a postulated model of the core-mantle boundary, one would be able to expect the effects of the corresponding transfer functions on various core phases. Conversely, by examining these effects, it becomes simple to select the most desirable kind of core phase data, in terms of wave types, recording frequency bands, and epicentral distances, which would lead to the best experimental results.

In Figures 2, and 3, comparisons are made between several transfer functions computed for the five models given in Table 1 . These curves, plotted at fixed incident 
TABLE 1

Models of Core-Mantle Boundary

\begin{tabular}{|c|c|c|c|c|c|}
\hline $\begin{array}{l}\text { Model } \\
\text { No. }\end{array}$ & $\begin{array}{c}\text { Layer } \\
\text { No. }\end{array}$ & $\begin{array}{c}\text { Thickness } \\
\mathrm{km}\end{array}$ & $\begin{array}{c}\alpha \\
\mathrm{km} / \mathrm{sec}\end{array}$ & $\begin{array}{c}B \\
\mathrm{~km} / \mathrm{sec}\end{array}$ & $\stackrel{p}{\mathrm{gr} / \mathrm{cm}^{3}}$ \\
\hline \multirow[t]{4}{*}{1} & 0 & $\infty$ & 8.040 & 0.000 & 10.060 \\
\hline & 1 & 20.00 & 13.680 & 7.200 & 5.355 \\
\hline & 2 & 80.00 & 13.700 & 7.225 & 5.325 \\
\hline & 3 & $\infty$ & 13.700 & 7.250 & 5.300 \\
\hline \multirow[t]{6}{*}{2} & 0 & $\infty$ & 8.150 & 0.000 & 9.400 \\
\hline & 1 & 18.00 & 13.720 & 7.195 & 5.675 \\
\hline & 2 & 20.00 & 13.710 & 7.200 & 5.665 \\
\hline & 3 & 20.00 & 13.700 & 7.205 & 5.655 \\
\hline & 4 & 20.00 & 13.690 & 7.215 & 5.645 \\
\hline & 5 & $\infty$ & 13.680 & 7.220 & 5.640 \\
\hline \multirow[t]{5}{*}{3} & 0 & $\infty$ & 8.150 & 0.000 & 9.400 \\
\hline & 1 & 11.00 & 10.200 & 5.200 & 6.200 \\
\hline & 2 & 13.00 & 11.600 & 6.100 & 5.670 \\
\hline & 3 & 12.00 & 13.000 & 6.840 & 5.660 \\
\hline & 4 & $\infty$ & 13.690 & 7.210 & 5.650 \\
\hline \multirow[t]{3}{*}{4} & 0 & $\infty$ & 8.300 & 0.000 & 9.500 \\
\hline & 1 & 30.00 & 10.000 & 2.800 & 6.700 \\
\hline & 2 & $\infty$ & 13.600 & 7.500 & 5.500 \\
\hline \multirow[t]{3}{*}{5} & 0 & $\infty$ & 8.300 & 0.000 & 9.500 \\
\hline & 1 & 100,00 & 13.300 & 4.800 & 6.700 \\
\hline & 2 & $\infty$ & 13.600 & 7.500 & 5.500 \\
\hline
\end{tabular}

† The interface between the zeroth and the first layers corresponds to a depth of $2898 \mathrm{~km}$. The model number refers to:

(1) Gutenberg-Bullard I (Landisman et al, 1965)

(2) Standard model (Dorman et al, 1966)

(3) Model R 1 (Dorman et al, 1966)

(4) Model 94 (Phinney and Alexander, 1966)

(5) Model 81 (Phinney and Alexander, 1966)

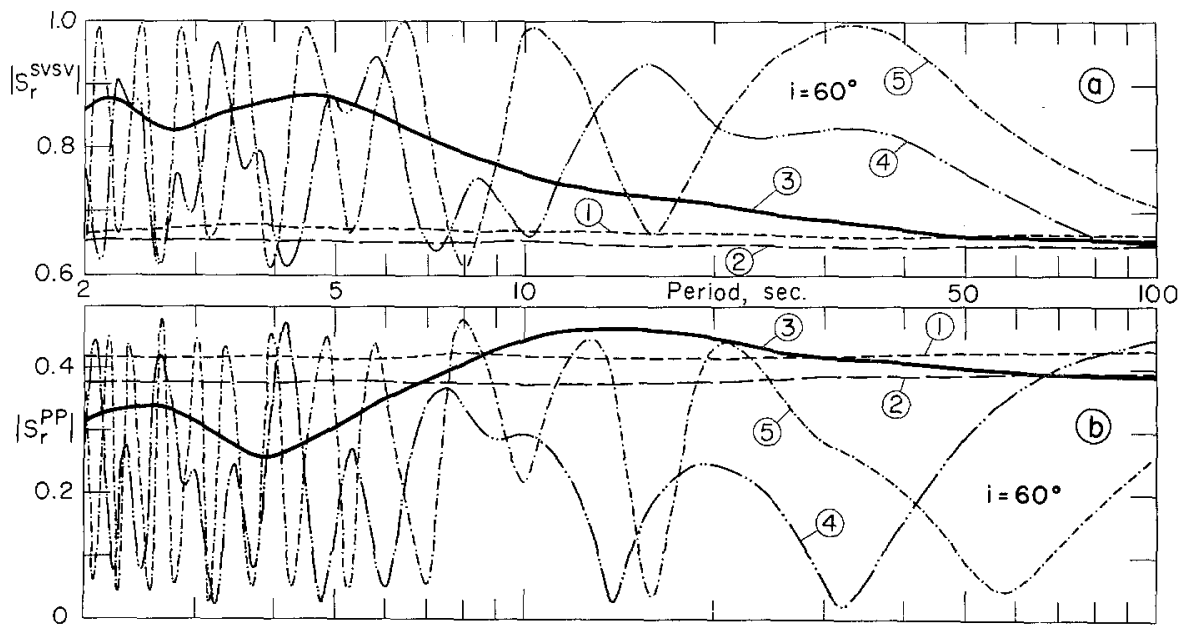

FIG. 2. Comparisons between the five core-mantle boundary models given in Table 1. (a) Amplitude ratio for the reflected $S$ wave due to an $S$-wave incident in the mantle against the core. (b) Amplitude ratio for the reflected $P$ wave due to a $P$-wave incident in the mantle against the core. 

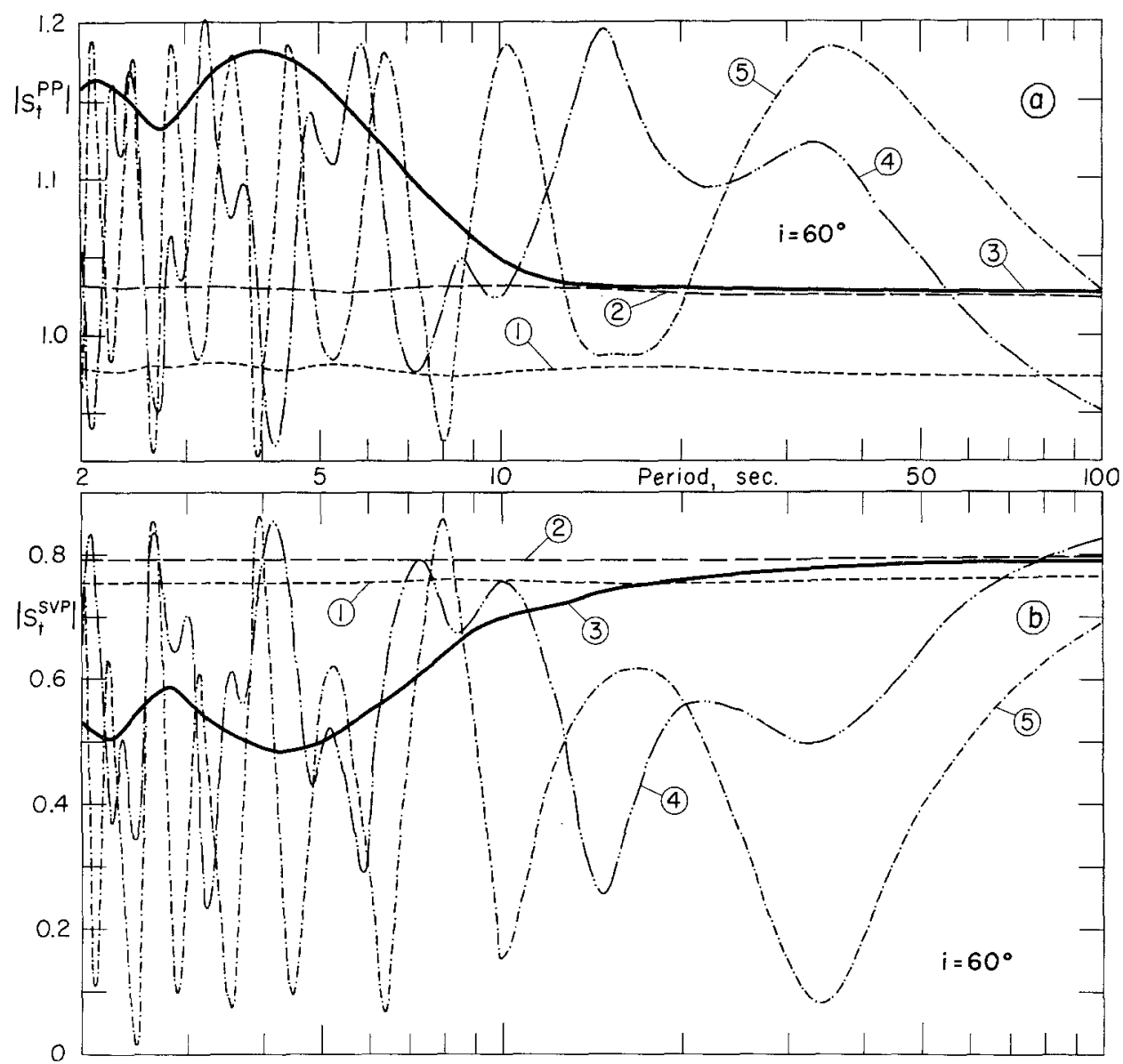

FIG. 3. Comparisons between the five core-mantle boundary models given in Table 1 . (a) Amplitude ratio for the transmitted $P$ wave due to a $P$-wave incident in the mantle against the core. (b) Amplitude ratio for the transmitted $P$ wave due to an $S$-wave incident in the mantle against the core.

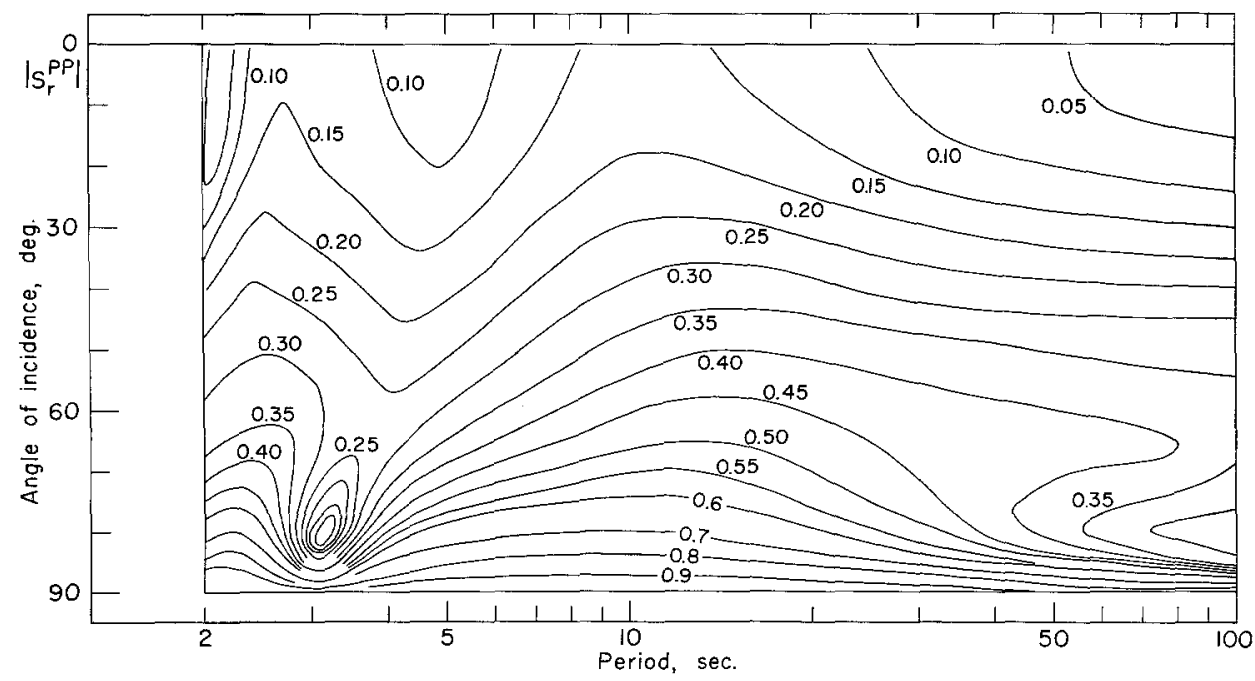

FIG. 4. Amplitude ratio for the reflected $P$ wave due to a $P$-wave incident in the mantle against the core. 
angle $i=60^{\circ}$, indicate the degree of distortion a wave signal would have suffered from reflecting off or transmitting through such a layered model. Clearly, the function $S_{r}{ }^{S V, V}$ is associated with $S c S$, likewise $S_{r}{ }^{P P}$ with $P c P, S_{t}{ }^{P P}$ with $P K P$, and

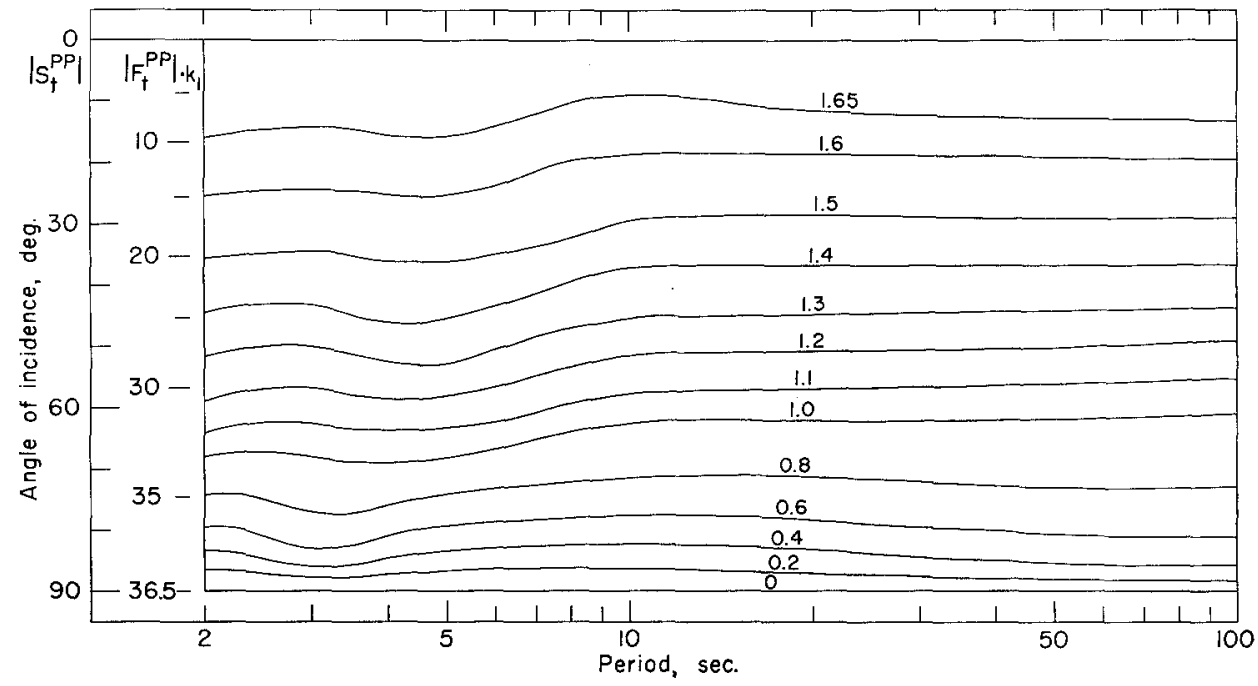

FIG. 5. Amplitude ratio for the transmitted $P$ wave due to a $P$-wave incident in the mantle (core) against the core (mantle). $k_{1}=\left(\rho_{n} \alpha^{4} r_{\alpha_{n}}\right) /\left(\rho_{0} \alpha_{0}^{4} r_{a_{0}}\right)$, its numerical values are shown in Figure 9.

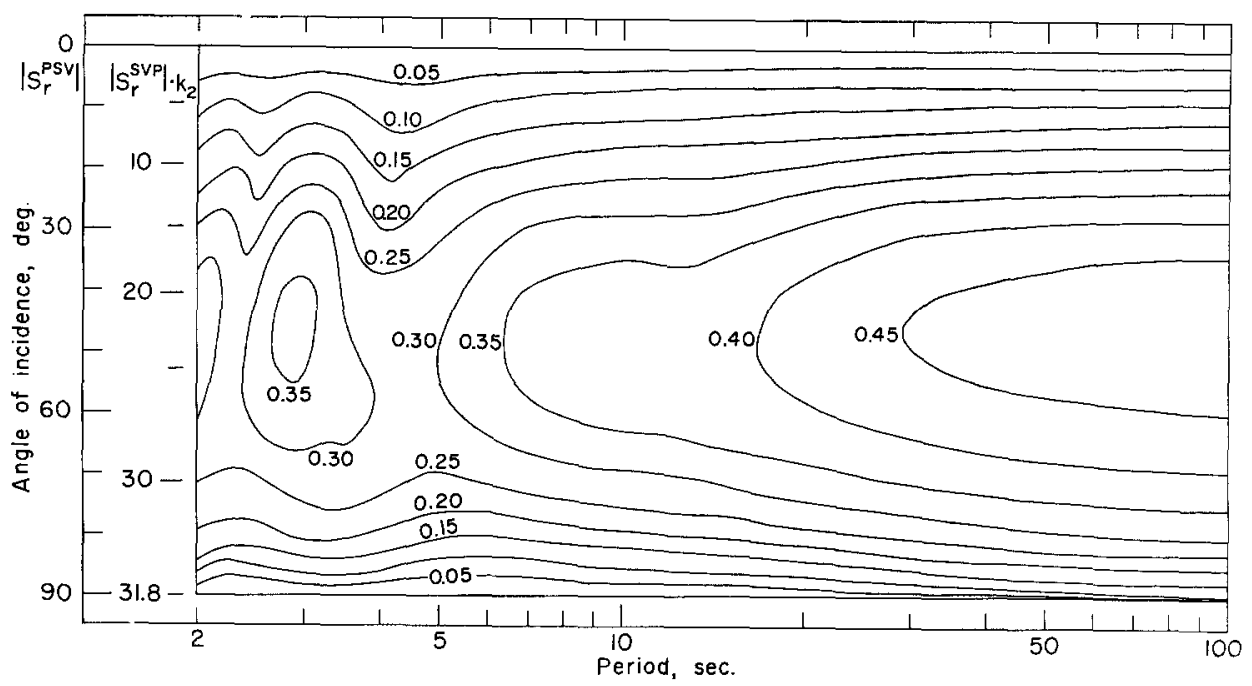

Frg. 6. Amplitude ratio for the reflected $S(P)$ wave due to a $P(S)$-wave incident in the mantle against the core. $k_{2}=\left(\alpha_{n}{ }^{4} r_{\alpha_{n}}\right) /\left(4 \beta_{n}{ }^{4} r_{\beta_{n}}\right)$, its numerical values are shown in Figure 9 .

$S_{t}{ }^{S P}$ with $S K S$, etc. Over the entire plotted period range, these functional forms for model 1 and model 2 hardly differ from those for the case of two half-spaces in contact. To discriminate model 1 from model 2, one would have to go to much higher frequencies and shallower incident angles. Between 10 and 100 seconds period range, 
it is difficult with the present capability of the long-period recording instruments to separate model 3 from the previous two models. However, this separation becomes possible for periods shorter than ten seconds. Among wave types, $P c P$ appears to be

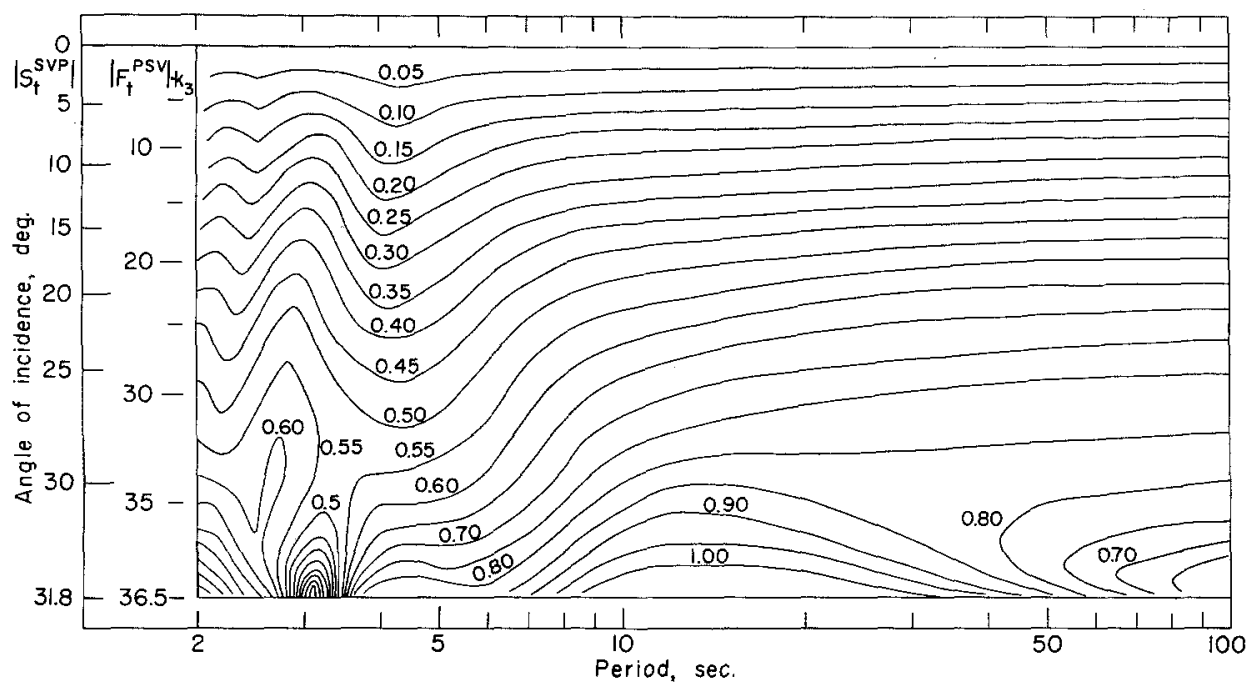

FIG. 7. Amplitude ratio for the transmitted $P(S)$ wave due to an $S(P)$-wave incident in the mantle (core) against the core (mantle). $k_{2}=\left(2 \rho_{n} \beta_{n}{ }^{4} r_{\beta_{n}}\right) /\left(\rho_{0} \alpha_{0}{ }^{4} r_{\alpha_{0}}\right)$, its numerical values are shown in Figure 9.

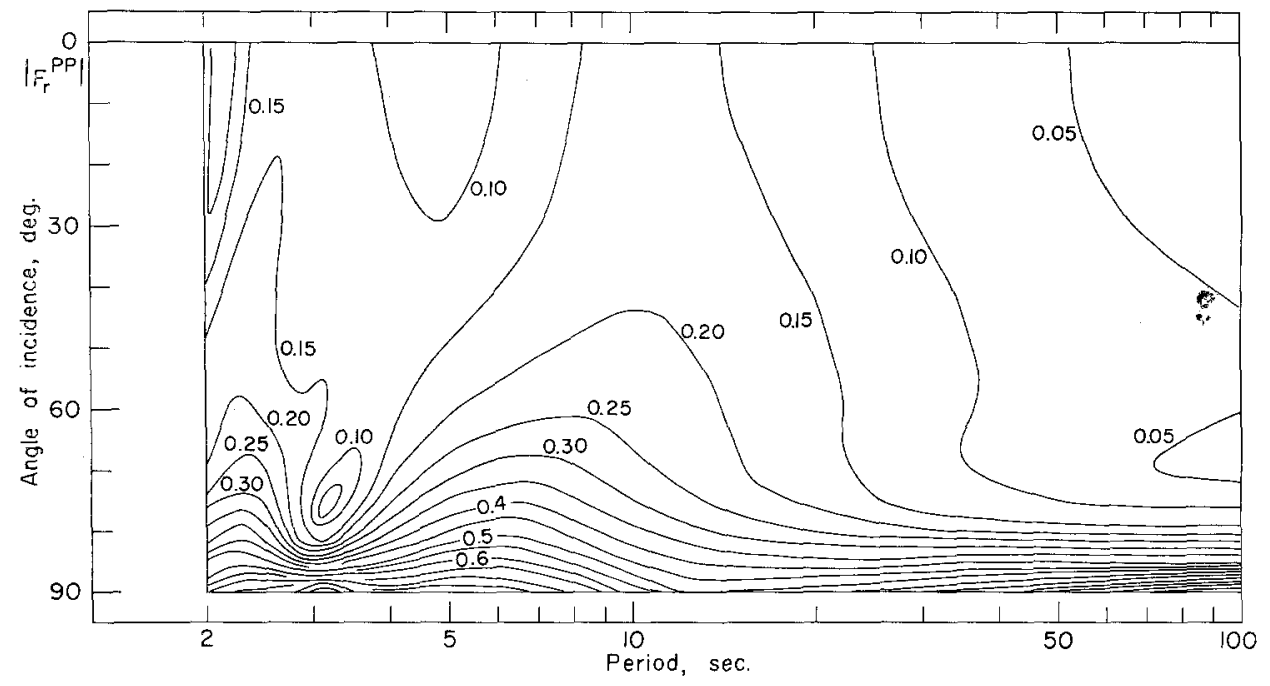

Fig. 8. Amplitude ratio for the reflected $P$ wave due to a $P$-wave incident in the core against the mantle.

more desirable for this purpose, because the function $S_{r}{ }^{P P}$ has the largest variation ( $\sim 40 \%$ ) over the period range of 2 to 10 seconds. All amplitude functions for model 4 and model 5 have distinctive characteristics. Therefore, all core phases except those with steep incident angles can be conveniently used to find out whether the 
true model of the core mantle boundary is closer to model 4 or model 5 , or instead to the first three models. Also revealed by these figures are the larger variations of $S_{r}{ }^{P P}$ and $S_{t}{ }^{S V P}(>40 \%)$ as compared to those of $S_{r}{ }^{S V S V}$ and $S_{t}{ }^{P P}(<20 \%)$. These results imply that $P c P$ and $S K S$ are probably more sensitive to the core-mantle boundary structure while $S c S$ and $P K P$ are more useful in attenuation studies.

To show the variations of these transfer functions with changing incident angles, we present in Figures $4,5,6,7$, and 8 , the functions $S_{r}{ }^{P P}, S_{t}{ }^{P P}, F_{t}{ }^{P P}, S_{r}{ }^{P S V}, S_{r}{ }^{S V P}$, $S_{t}{ }^{S V P}, F_{t}{ }^{P S V}$ and $F_{r}{ }^{P P}$ in the form of contour maps. They are computed based on model 3 , the associated numerical values of $k_{1}, k_{2}$, and $k_{3}$ are presented in Figure 9. The function $S_{r}{ }^{S V S V}$ is not shown here but it can be obtained easily from these

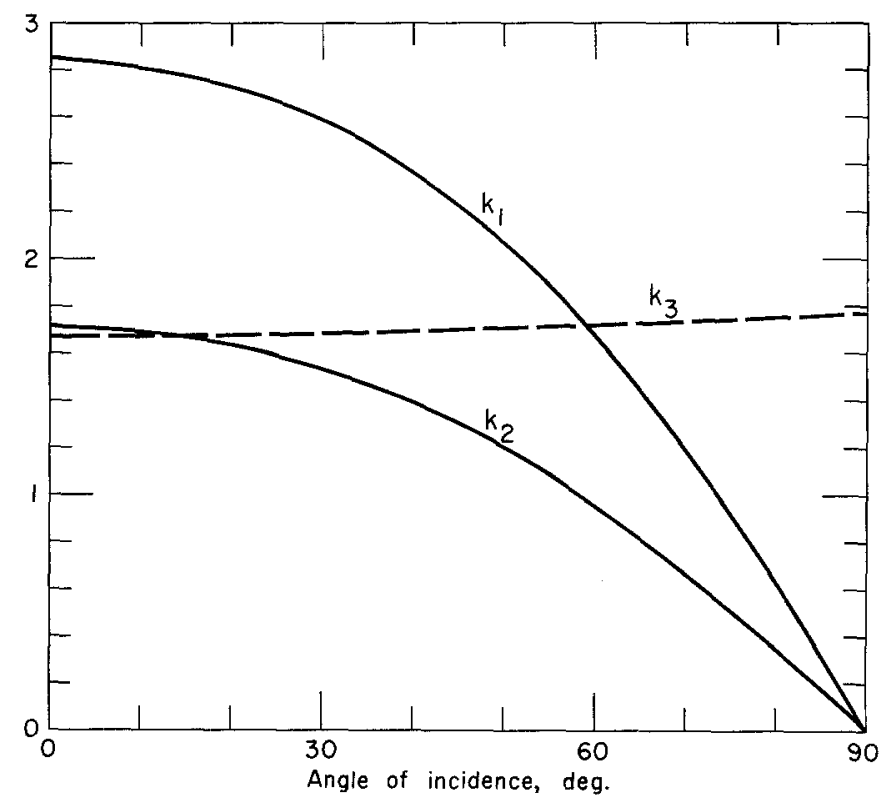

?

FIG. 9. The conversion constants for Model 3.

figures through the simple relations (32) and (33). These contour maps present a comprehensive picture that is useful in designing experiments. Regions with rough topography can quickly be located which, with reference to travel-time charts and instrumental response curves, simply point out the optimal choice in wave type, epicentral distances and recording instruments for a more effective investigation on the structure. By the same token but with different emphasis, regions with relatively flat topography indicate that the effects of wave transmission and reflection are small and may be neglected under some circumstances.

$A s$ it is expected by the scale law of the wavelength, the topography becomes progressively complicated with increasing incident angle and frequency. When the frequency approaches zero, the values of each function approach the right limits which are equivalent to the case of a fluid and a solid half spaces in contact. 
Appendix A

ON The Fuutd Layer Matrix

For solid layers, the matrix $E_{m}$ relating the motion-stress vector $\left(\dot{u}_{m} / c, \dot{w}_{m} / c\right.$, $\left.\sigma_{m}, \tau_{m}\right)$ with the vector $\left(\Delta_{m}{ }^{\prime}+\Delta_{m}^{\prime \prime}, \Delta_{m}{ }^{\prime}-\Delta_{m}{ }^{\prime \prime}, \omega_{m}{ }^{\prime}-\omega_{m}^{\prime \prime}, \omega_{m}{ }^{\prime}+\omega_{m}^{\prime \prime}\right)$ across the $(m-1)^{\text {th }}$ interface, and the matrix $D_{m}$, connecting the two vectors across the $m^{\text {th }}$ layer, are unambiguously given by Haskell (1953, equations 12 and 14 ). However, as the theory was extended to include fluid layers by setting $\beta_{m}=0$, it was found that the matrix $E_{m}$ becomes singular. This led Haskell to define an effective inverse $E_{m}$ (expressed by $F_{m}^{-1}$ in equation 6.1 ) such that the layered matrix $a_{m}$ (equation 6.3) can be constructed. Haskell (see Dorman, 1962) later pointed out that on setting $\beta_{m}=0$, the matrices $E_{m}$ and $D_{m}$ lead to overspecified boundary conditions, because only vertical motion and normal stress are continuous across a fluid interface. Using these two boundary equations, Dorman (1962) derived a $2 \times$ 2 matrix $l_{m}$, for fluid layers which properly accounts for the boundary conditions.

It is possible to write a $4 \times 4$ matrix for a fluid layer such that, for a sequence of fluid and solid interbeds, dimensions of the layer matrices are consistant throughout in the matrix formulation. In addition to being formally desirable, it also offers obvious convenience in numerical programming.

Since only vertical motion $\dot{w} / c$ and normal stress $\sigma$ are continuous for a fluid layer, we can think of the two-dimensional motion-stress vector $(\dot{w} / c, \sigma)$ and its associated dilation vector $\left(\Delta^{\prime}+\Delta^{\prime \prime}, \Delta^{\prime}-\Delta^{\prime \prime}\right)$ as two degenerated four-dimensional vectors $(0, \dot{w} / c, \sigma, 0)$ and $\left(\Delta^{\prime}+\Delta^{\prime}, \Delta^{\prime}-\Delta^{\prime \prime}, 0,0\right)$. Our problem then is to find the correct transformations, or matrix operators, which relate the two degenerated vectors across a fluid interface or a fluid layer.

The boundary conditions for the $(m-1)^{\text {th }}$ interface are

$$
\begin{gathered}
\dot{w}_{m-1} / c=-\left(\alpha_{m} / c\right)^{2} r_{\alpha_{m}}\left(\Delta_{m}{ }^{\prime}-\Delta_{m}{ }^{\prime \prime}\right) \\
\sigma_{m-1}=\rho_{m} \alpha_{m}{ }^{2}\left(\Delta_{m}{ }^{\prime}+\Delta_{m}{ }^{\prime \prime}\right) .
\end{gathered}
$$

Corresponding to $E_{m}$ for a solid interface, define for a fluid interface a $4 \times 4$ matrix $E_{m}{ }^{\prime}$ such that

$$
\left[\begin{array}{c}
0 \\
\frac{\dot{w}_{m-1}}{c} \\
\sigma_{m} \\
0
\end{array}\right]=E_{m}{ }^{\prime}\left[\begin{array}{c}
{\Delta_{m}}^{\prime}+\Delta_{m}{ }^{\prime \prime} \\
\Delta_{m}{ }^{\prime}-\Delta_{m}^{\prime \prime} \\
0 \\
0
\end{array}\right]
$$

and 


$$
E_{m}^{\prime}=\left[\begin{array}{cccc}
0 & 0 & b_{11} & b_{12} \\
0 & -\left(\alpha_{m} / c\right)^{2} r_{\alpha_{m}} & b_{13} & b_{14} \\
\rho_{m} \alpha_{m}{ }^{2} & 0 & b_{15} & b_{16} \\
0 & 0 & b_{17} & b_{18}
\end{array}\right]
$$

where $b_{i j}(i=1,2,3,4 ; j=1,2, \cdots 8)$ are certain functions to be determined. The inverse transformations of (A-1)

$$
\begin{aligned}
& {\Delta_{m}}^{\prime}+\Delta_{m}{ }^{\prime \prime}=\sigma_{m-1} / \rho_{m} \alpha_{m}^{2} \\
& \Delta_{m}{ }^{\prime}-\Delta_{m}{ }^{\prime \prime}=\left(c / \alpha_{m}\right)^{2} r_{\alpha_{m}}^{-1} \dot{w}_{m-1} / c
\end{aligned}
$$

implies that $E_{m}{ }^{\prime}$ cannot be singular. Its inverse $\left(E_{m}{ }^{\prime}\right)^{-1}$ can be written by

$$
\left(E_{m}{ }^{\prime}\right)^{-1}=\left[\begin{array}{cccc}
b_{21} & 0 & \left(\rho_{m} \alpha_{m}{ }^{2}\right)^{-1} & b_{22} \\
b_{23} & -\left(c / \alpha_{m}\right)^{2} r_{\alpha_{m}}^{-1} & 0 & b_{24} \\
b_{25} & 0 & 0 & b_{26} \\
b_{27} & 0 & 0 & b_{28}
\end{array}\right]
$$

Across the $n^{\text {th }}$ layer, the transformations are

$$
\begin{aligned}
\dot{w}_{m} / c & =-\left(\alpha_{m} / c\right)^{2} r_{\alpha_{m}}\left[-i\left(\Delta_{m}{ }^{\prime}+\Delta_{m}{ }^{\prime \prime}\right) \sin P_{m}+\left(\Delta_{m}{ }^{\prime}-\Delta_{m}{ }^{\prime \prime}\right) \cos P_{m}\right] \\
\sigma_{m} & =\rho_{m} \alpha_{m}{ }^{2}\left[\left(\Delta_{m}{ }^{\prime}+\Delta_{m}{ }^{\prime \prime}\right) \cos P_{m}-i\left({\Delta_{m}}^{\prime}-\Delta_{m}{ }^{\prime \prime}\right) \sin P_{m}\right] .
\end{aligned}
$$

Then, corresponding to $D_{m}$ for a solid layer, define for a fluid layer a $4 \times 4$ matrix $D_{m}{ }^{\prime}$ such that

$$
\left[\begin{array}{c}
0 \\
\dot{w}_{m} / c \\
\sigma_{m} \\
0
\end{array}\right]=D_{m}{ }^{\prime}\left[\begin{array}{c}
\Delta_{m}{ }^{\prime}+\Delta_{m}^{\prime \prime} \\
\Delta_{m}{ }^{\prime}-\Delta_{m}^{\prime \prime} \\
0 \\
0
\end{array}\right]
$$

and

$$
D_{m}{ }^{\prime}=\left[\begin{array}{ccc}
0 & 0 & b_{31} b_{32} \\
i\left(\alpha_{m} / c\right)^{2} r_{\alpha_{m}} \sin P_{m} & -\left(\alpha_{m} / c\right)^{2} r_{\alpha_{m}} \cos P_{m} & b_{33} b_{34} \\
\rho_{m} \alpha_{m}{ }^{2} \cos P_{m} & -i \rho_{m} \alpha_{m}{ }^{2} \sin P_{m} & b_{35} b_{36} \\
0 & 0 & b_{32} b_{38}
\end{array}\right]
$$


Moreover, a $4 \times 4$ fluid layer matrix $a_{m}{ }^{\prime}=D_{m}{ }^{\prime}\left(E_{m}{ }^{\prime}\right)^{-1}$ can be defined which governs the transformations of the motion-stress vector between layers

$$
\left[\begin{array}{c}
0 \\
\dot{w}_{m} / c \\
\sigma_{m} \\
0
\end{array}\right]=a_{m}^{\prime}\left[\begin{array}{c}
0 \\
\dot{w}_{m-1} / c \\
\sigma_{m-1} \\
0
\end{array}\right]
$$

by

$$
a_{, n}{ }^{\prime}=\left[\begin{array}{cccc}
b_{41} & 0 & 0 & b_{45} \\
b_{42} & \cos P_{m} & i r_{\alpha_{m}}\left(\rho_{m} c^{2}\right)^{-1} \sin P_{m} & b_{45} \\
b_{43} & i \rho_{m} c^{2} r_{\alpha_{m}}^{-1} \sin P_{m} & \cos P_{m} & b_{42} \\
b_{44} & 0 & 0 & b_{48}
\end{array}\right] .
$$

Since by definition

$$
\begin{gathered}
E_{m}{ }^{\prime}\left(E_{m}{ }^{\prime}\right)^{-1}=\left(E_{m}{ }^{\prime}\right)^{-1} E_{m}{ }^{\prime}=I \\
a_{m}{ }^{\prime}=D_{m}{ }^{\prime}\left(E_{m}{ }^{\prime}\right)^{-1}
\end{gathered}
$$

(A-11) and (A-12) still do not provide enough independent conditions to uniquely determine the $b_{i j}$ 's. We then further require that when the thickness of a layer approaches zero, the layer matrix $a_{m}{ }^{\prime}$ becomes an identity matrix. With this additional condition, it is found and can be verified by direct substitution that

$$
b_{12}=b_{17}=b_{26}=b_{27}=b_{32}=b_{37}=b_{41}=b_{48}=1
$$

and all other $b_{i j}$ 's are zero.

We, therefore, obtain the following $4 \times 4$ matrices for a fluid layer

$$
\begin{aligned}
E_{m}{ }^{\prime} & =\left[\begin{array}{cccc}
0 & 0 & 0 & 1 \\
0 & -\left(\alpha_{m} / c\right)^{2} r_{\alpha_{m}} & 0 & 0 \\
\rho_{m} \alpha_{m}{ }^{2} & 0 & 0 & 0 \\
0 & 0 & 1 & 0
\end{array}\right] \\
\left(E_{m}{ }^{\prime}\right)^{-1} & =\left[\begin{array}{cccc}
0 & 0 & \left(\rho_{m} \alpha_{m}{ }^{2}\right)^{-1} & 0 \\
0 & -\left(c / \alpha_{m}\right)^{2} r_{\alpha_{m}}{ }^{-1} & 0 & 0 \\
0 & 0 & 0 & 1 \\
1 & 0 & 0 & 0
\end{array}\right]
\end{aligned}
$$




$$
D_{m}{ }^{\prime}=\left[\begin{array}{cccc}
0 & 0 & 0 & 1 \\
i\left(\alpha_{m} / c\right)^{2} r_{\alpha_{m}} \sin P_{m} & -\left(\alpha_{m} / c\right)^{2} r_{\alpha_{m}} \cos P_{m} & 0 & 0 \\
\rho_{m} \alpha_{m}{ }^{2} \cos P_{m} & -i \rho_{m} \alpha_{m}{ }^{2} \sin P_{m} & 0 & 0 \\
0 & 0 & 1 & 0
\end{array}\right]
$$

and

$$
a_{m}{ }^{\prime}=\left[\begin{array}{cccc}
1 & 0 & 0 & 0 \\
0 & \cos P_{m} & i r_{\alpha_{m}}\left(\rho_{m} c\right)^{-1} \sin P_{m} & 0 \\
0 & i \rho_{m} c^{2} r_{\alpha_{m}}^{-1} \sin P_{m} & \cos P_{m} & 0 \\
0 & 0 & 0 & 1
\end{array}\right]
$$

\section{Appendix B}

In this appendix we shall give proof to the relations in equation (33). We first write

$$
E_{n}^{-1}=\left(\eta_{i j}\right)=\left[\begin{array}{cccc}
-2\left(\beta_{n} / \alpha_{n}\right)^{2} & 0 & \left(\rho_{n} \alpha_{n}{ }^{2}\right)^{-1} & 0 \\
0 & c^{2}\left(\gamma_{n-1}\right) / \alpha_{n}{ }^{2} r_{\alpha_{n}} & 0 & \left(\rho_{n} \alpha_{n}{ }^{2} r_{\alpha_{n}}\right)^{-1} \\
\left(\gamma_{n-1}\right) / \gamma_{n} r_{\beta_{n}} & 0 & -\left(\rho_{n} c^{2} \gamma_{n} r_{\beta_{n}}\right)^{-1} & 0 \\
0 & 1 & 0 & \left(\rho_{n} c^{2} \gamma_{n}\right)^{-1}
\end{array}\right](\mathrm{B}-1)
$$

and note that

$$
\begin{aligned}
\eta_{22} \eta_{: 3} & =-\eta_{31} \eta_{24} \\
\eta_{13} & =-\eta_{11} \eta_{44} .
\end{aligned}
$$

By virtue of the law of energy conservation, the elements of the matrix $A=\left(A_{i j}\right)$ obey the following identities (Haskell, 1962)

$$
\begin{aligned}
& A_{11} A_{42}-A_{21} A_{32}+A_{31} A_{22}-A_{41} A_{12} \equiv 0 \\
& A_{21} A_{33}-A_{11} A_{43}+A_{13} A_{41}-A_{23} A_{31} \equiv 0 \\
& A_{12} A_{44}-A_{22} A_{34}+A_{32} A_{24}-A_{42} A_{14} \equiv 0 \\
& A_{13} A_{44}-A_{23} A_{34}+A_{24} A_{33}-A_{14} A_{43} \equiv 0 \\
& A_{11} A_{44}-A_{21} A_{34}+A_{24} A_{31}-A_{14} A_{41} \equiv 1 \\
& A_{22} A_{33}-A_{12} A_{43}+A_{13} A_{42}-A_{23} A_{32} \equiv 1 .
\end{aligned}
$$


The matrix $J=\left(J_{i j}\right)$, the elements of which make up our transfer functions, is related to the matrix $A$ by,

$$
J_{i j}=\eta_{i k} A_{k j}
$$

(1) To prove the first relation of (33), it is sufficient to show that

$$
\begin{aligned}
\frac{c^{2}}{\rho_{n} \alpha_{n}{ }^{4} r_{\alpha n}}\left(J_{31}-J_{41}\right) & -\left[\left(J_{31}-J_{41}\right)\left(J_{12} J_{23}-J_{13} J_{22}\right)\right. \\
+ & \left(J_{32}-J_{42}\right)\left(J_{13} J_{21}-J_{11} J_{23}\right) \\
+ & \left.\left(J_{33}-J_{43}\right)\left(J_{11} J_{22}-J_{12} J_{21}\right)\right]=0 .
\end{aligned}
$$

By (B-1), we have

$$
\frac{c^{2}}{\rho_{n} \frac{\alpha_{n}{ }^{4} r_{\alpha_{n}}}{4}}=-\eta_{11} \eta_{24}-\eta_{13} \eta_{22} .
$$

Denoting by $L_{1}$ the left-hand side of (B-11) and making use of (B-9), (B-10) and (B-12), we find

$$
\begin{aligned}
L_{1}= & \left(\eta_{31} A_{11}+\eta_{33} A_{31}-A_{21}-\eta_{44} A_{41}\right)\left[\eta_{11} \eta_{24}\left(A_{22} A_{33}-A_{23} A_{32}\right)\right. \\
& +\eta_{13} \eta_{22}\left(A_{13} A_{42}-A_{12} A_{43}\right)+\eta_{11} \eta_{22}\left(A_{12} A_{23}-A_{13} A_{22}\right) \\
& \left.+\eta_{13} \eta_{24}\left(A_{32} A_{43}-A_{33} A_{42}\right)\right]+\left(\eta_{31} A_{12}+\eta_{33} A_{32}-A_{22}-\eta_{44} A_{42}\right) \\
& \cdot\left[\left(\eta_{11} A_{13}+\eta_{13} A_{33}\right)\left(\eta_{22} A_{21}+\eta_{24} A_{41}\right)-\left(\eta_{11} A_{11}+\eta_{13} A_{31}\right)\left(\eta_{22} A_{23}\right.\right. \\
& \left.\left.+\eta_{24} A_{43}\right)\right]+\left(\eta_{31} A_{13}+\eta_{33} A_{33}-A_{23}-\eta_{44} A_{43}\right)\left[\left(\eta_{11} A_{11}+\eta_{13} A_{31}\right)\right. \\
& \left.\cdot\left(\eta_{22} A_{22}+\eta_{24} A_{42}\right)-\left(\eta_{11} A_{12}+\eta_{13} A_{32}\right)\left(\eta_{22} A_{21}+\eta_{24} A_{41}\right)\right] .
\end{aligned}
$$

Expanding the above expression and gathering terms, it is found that the coefficients of $\eta_{11} \eta_{22} \eta_{31}, \eta_{11} \eta_{22}, \eta_{13} \eta_{24} \eta_{33}$, and $\eta_{13} \eta_{24} \eta_{44}$ vanish identically. Using the relations (B-2), (B-3), (B-4), and (B-5), the remaining eight terms, denoted by $I_{1}$ through $I_{8}$, can be calculated as follows,

$$
\begin{aligned}
I_{1}=\eta_{11} \eta_{22} \eta_{33}\left[A_{12} A_{23} A_{31}\right. & -A_{12} A_{21} A_{33}+A_{11} A_{12} A_{43} \\
& \left.+A_{13}\left(A_{21} A_{32}-A_{22} A_{31}-A_{11} A_{42}\right)\right] \\
= & \eta_{11} \eta_{12} \eta_{13} A_{12}\left(A_{23} A_{31}-A_{21} A_{33}+A_{11} A_{43}-A_{13} A_{41}\right) \\
= & 0
\end{aligned}
$$




$$
\begin{aligned}
I_{2}= & \eta_{11} \eta_{22} \eta_{44}\left[A_{11} A_{23} A_{42}-A_{23} A_{32} A_{21}-A_{23} A_{12} A_{41}\right. \\
& \left.\quad-A_{22}\left(A_{11} A_{43}-A_{13} A_{41}-A_{33} A_{21}\right)\right] \\
= & \eta_{11} \eta_{22} \eta_{44} A_{23}\left(A_{11} A_{42}-A_{32} A_{21}+A_{22} A_{31}-A_{12} A_{41}\right) \\
= & 0 \\
I_{3}= & \eta_{11} \eta_{24} \eta_{33}\left[A_{13} A_{41} A_{32}-A_{11} A_{43} A_{32}-A_{23} A_{32} A_{31}-A_{33}\left(A_{12} A_{41}-A_{22} A_{31}\right.\right. \\
& \left.\left.\quad-A_{11} A_{42}\right)\right] \\
= & \eta_{11} \eta_{24} \eta_{33} A_{32}\left(A_{13} A_{41}-A_{11} A_{43}-A_{23} A_{31}+A_{33} A_{21}\right) \\
= & 0 \\
I_{4}= & \eta_{11} \eta_{24}\left[A_{12} A_{41} A_{23}-A_{11} A_{42} A_{23}+A_{23} A_{32} A_{21}+A_{22}\left(A_{11} A_{43}-A_{13} A_{41}\right.\right. \\
= & \left.\quad-A_{13} \eta_{22} \eta_{33} A_{32}\left(A_{13} A_{41}-A_{31} A_{22}-A_{23} A_{31}+A_{21} A_{33}-A_{41}+A_{11} A_{43}\right)\right]
\end{aligned}
$$




$$
=0
$$

$$
\begin{aligned}
I_{8}=\eta_{13} \eta_{22} \eta_{41}\left[A_{32} A_{21} A_{43}-\right. & A_{31} A_{22} A_{43}+A_{12} A_{43} A_{41}+A_{42}\left(A_{31} A_{23}\right. \\
& \left.\left.\quad-A_{21} A_{33}-A_{13} A_{41}\right)\right] \\
= & \eta_{13} \eta_{22} \eta_{44} A_{43}\left(A_{32} A_{21}-A_{22} A_{31}+A_{12} A_{41}-A_{11} A_{42}\right) \\
= & 0 .
\end{aligned}
$$

This concludes that $L_{1}=0$ and the equality of $E_{S_{t}}^{P P}(c)$ and $E_{F_{i}}^{P P}(c)$ is established.

(2) The second relation of (33) can be established by showing that

$$
\begin{aligned}
4 \beta_{n}{ }^{4} r_{\beta_{n}}\left[\left(J_{31} J_{42}-\right.\right. & \left.\left.J_{32} J_{41}\right) p+\left(J_{31} J_{43}-J_{33} J_{41}\right) q\right] \\
& +\alpha_{n}{ }^{4} r_{\alpha_{n}}\left[\left(J_{11} J_{22}-J_{12} J_{21}\right) p+\left(J_{11} J_{23}-J_{13} J_{21}\right) q\right]=0 .
\end{aligned}
$$

Since the equality

$$
\left(J_{32} J_{41}-J_{31} J_{42}\right)=-\left(\alpha_{n}{ }^{4} r_{\alpha_{n}} / 4 \beta_{n} r_{\beta_{n}}\right)\left(J_{12} J_{21}-J_{11} J_{22}\right)
$$

has been shown by Haskell (1962), our problem becomes one of showing

$$
4 \beta_{n}{ }^{4} r_{\beta_{n}}\left(J_{31} J_{43}-J_{33} J_{41}\right)-\alpha_{n}{ }^{4}{ }^{2}{ }_{\alpha_{n}}\left(J_{11} J_{23}-J_{13} J_{21}\right)=0 .
$$

Denoting the left-hand side of (B-17) by $L_{2}$ and writing it out explicitly in terms of the elements of $A$, we have

$$
\begin{aligned}
L_{2}= & 4 \beta_{n}{ }^{4} r_{\beta_{n}}\left[\eta_{31}\left(A_{11} A_{23}-A_{13} A_{21}\right)+\eta_{31} \eta_{44}\left(A_{11} A_{43}-A_{13} A_{41}\right)\right. \\
& \left.+\eta_{33}\left(A_{31} A_{23}-A_{33} A_{21}\right)+\eta_{33} \eta_{44}\left(A_{31} A_{43}-A_{33} A_{41}\right)\right] \\
& +\alpha_{n}{ }^{4} r_{\alpha_{n}}\left[\eta_{11} \eta_{22}\left(A_{11} A_{23}-A_{13} A_{21}\right)+\eta_{11} \eta_{24}\left(A_{11} A_{43}-A_{13} A_{41}\right)\right. \\
& \left.+\eta_{13} \eta_{22}\left(A_{31} A_{23}-A_{33} A_{21}\right)+\eta_{13} \eta_{24}\left(A_{31} A_{43}-A_{33} A_{41}\right)\right] .
\end{aligned}
$$

By use of the following relations

$$
4 \beta_{n}{ }^{4} r_{\beta_{n}} \cdot\left[\begin{array}{c}
\eta_{31} \\
\eta_{31} \eta_{34} \\
\eta_{33} \\
\eta_{33} \eta_{44}
\end{array}\right]=\alpha_{n}{ }^{4} r_{\alpha_{n}} \cdot\left[\begin{array}{cc}
-\eta_{11} & \eta_{22} \\
\eta_{13} & \eta_{22} \\
\eta_{11} & \eta_{24} \\
-\eta_{13} & \eta_{24}
\end{array}\right]
$$

we rearrange (B-18) and find 


$$
\begin{aligned}
L_{2}= & 4 \beta_{n}{ }^{4} \gamma_{\beta_{n}}\left[\eta_{31}\left(A_{11} A_{23}-A_{13} A_{21}-A_{11} A_{23}+A_{13} A_{21}\right)\right. \\
& +\eta_{31} \eta_{44}\left(A_{11} A_{43}-A_{13} A_{41}+A_{31} A_{23}-A_{33} A_{21}\right) \\
& +\eta_{33}\left(A_{31} A_{23}-A_{33} A_{21}+A_{11} A_{43}-A_{13} A_{41}\right) \\
& \left.+\eta_{33} \eta_{44}\left(A_{31} A_{43}-A_{33} A_{41}-A_{31} A_{43}+A_{33} A_{41}\right)\right] \\
= & 0
\end{aligned}
$$

from equation (B-5). Therefore, equations (B-16) and (B-20) imply that

$$
E_{S_{r}}^{P S V}(c)=E_{S_{r}}^{S V P}(c) .
$$

(3) The third relation of (33) is proved following precisely the same steps we have just detailed above in (1). We shall not present the lengthy algebra here, only note that a relation similar to $(\mathrm{B}-12)$

$$
\frac{c^{2}}{4 \rho_{n} \beta_{n}{ }^{4} r_{\beta_{n}}}=-\eta_{33}-\eta_{31} \eta_{44}
$$

is found to be useful.

\section{ACKNOWLEDGMENTS}

It is a pleasure to thank Professor Don Anderson for reading the manuscript and offering several helpful suggestions. Assistance of Messrs. Laszlo Lenches and John Nordquist in the preparation of graphical materials is acknowledged.

This research was supported by the Air Force Office of Scientific Researeh, Office of Aerospace Research, United States Air Force, under AFOSR contract number AF-49(638)-1337.

\section{REFERENCES}

Alexander, S. S. and R. A. Phinney (1966). A study of core-mantle boundary using $P$-wave diffracted by the earth's core, J. Geophys. Res. 71, 5943-5958.

Bullen, K. E. (1963). An index of degree of chemical inhomogeneity in the earth, Geophys. $J$. $7,584-592$.

Dorman, J. (1962). Period equation for waves of Rayleigh type on a layered, liquid-solid halfspace, Bull. Seism. Soc. Am. 52, 389-397.

Dorman, J., J. Ewing and L. E. Alsop (1965). Oscillations of the earth: new core-mantle boundary model based on low order free vibrations, Proc. Nat. Acad. Sci. 54, 364-368.

Fernandez, L. M. (1965). The determination of erustal thickness from the spectrum of the $P$ wave, Doctoral Dissertation, St. Louis University.

Gutenberg, B. (1959). Physics of the Earth's Interior, p. 95, Academic Press, New York.

Haskell, N. A. (1953). The dispersion of surface waves in multilayered media, Bull. Seism. Soc. $A m .43,17-34$.

Haskell, N. A. (1960). Crustal reflection of plane $S H$ waves, $J$. Geophys. Res. 65, 4147-4150.

Haskell, N. A. (1962). Crustal reflection of plane $P$ and $S V$ waves, J. Geophys. Res. 67, 47514767.

Kanamori, H. (1966). Spectrum of $P$ and $P c P$ in relation to the mantle-core boundary and attenuation in the mantle, $J$. Geophys. Res., 72, 559-571.

Landisman, M., Y. Satî and J. Nafe (1965). Free vibration of the earth and the properties of its deep interior regions, Part 1: Density, Geophys. J. 9, 469. 
Phinney, R. A. (1964). Structure of the earth's crust from spectral behavior of long-period body waves, $J$. Geophys. Res. 69, 2997-3017.

Phinney, R. A. and S. S. Alexander (1966). Diffraction of $P$ waves and the structure of the core mantle boundary, J. Geophys. Res. 71, 5959-5975.

SeIsmological Laboratory

California Institute of Technology

Pasadena, California

(Division of Geological Sciences Contribution No. 1430)

Manuseript received November 14, 1966. 